\title{
Propuesta de la matriz de atractividad - competitividad en función de las modalidades turísticas.
}

Atractiveness- competitiveness matrix using a touristic niche proposal.

Lic. Diana Llanes Rosales., ${ }^{1} \&$ MSc. Danay M. Bulnes Mann. ${ }^{2}$

\section{Resumen.}

La presente investigación propone a partir de la matriz de atractividad-competitividad una nueva matriz que vincula las variables originales y añade la "modalidad turística". Se analizó la metodología para la conformación de la matriz de atractividad-competitividad y las decisiones estratégicas a tener en cuenta en cada uno de los cuadrantes que la conforman. Se estudiaron las variables principales de la matriz original: la competitividad del destino y la atractividad del mercado, para establecer una siguiente etapa de análisis de la variable a incorporar, modalidad turística. Para ello se consultó a expertos en el tema, obteniendo los principales atributos que inciden en el potencial de desarrollo de las modalidades turísticas. Se aplicaron métodos estadísticos y matemáticos para la elaboración y conformación de la matriz resultante, la cual servirá de apoyo a analistas e investigadores en la planeación estratégica de destinos turísticos para la introducción de nuevas modalidades.

Palabras claves: Competitividad, atractividad, modalidad turística, matriz de atractividad-competitividad.

\section{Abstract.}

The present investigation pretends, from the atractiveness -competitiveness matrix a new and more developed one that relates the original variables and ads the variable "touristic niche". First analyzing the methodology, how it works, their use and strategic decision to take into account, followed by a full study of the new variable. In order to do so there were consult several experts in the field and also were applied statistics and mathematics

\footnotetext{
${ }^{1}$ Universidad de La Habana, Facultad de Turismo. La Habana, Cuba. dianallanes1995@gmail.com ${ }^{2}$ Universidad de La Habana, Facultad de Turismo. La Habana, Cuba. danay_bulnes@ @tur.uh.cu
} 
methods to obtain the information, this new tool pretends to help analysts and investigators in the field of strategic planning.

Keywords: Destiny competitiveness, market atractiveness, touristic niche, atractiveness -competitiveness matrix.

\section{Introducción}

El turismo, en las últimas décadas, ha ido adquiriendo una gran importancia y todo apunta a que en un futuro seguirá su ritmo ascendente, considerándose como un fenómeno estructural, cuyo protagonismo en la economía internacional y en la de muchos países, regiones y localidades sea cada vez mayor. (Barroso y Flores 2006). Este significativo crecimiento en el número de destinos turísticos va a provocar un importante incremento de la competencia entre los mismos. De acuerdo a Dwyer y Kim (2003), para tener éxito en el mercado es necesario que un destino turístico (entendido como una región geográfica delimitada) asegure que los atractivos generales y todas las experiencias que ofrece a los visitantes sean superiores a las que ofrecen los otros destinos. Lo que en cierta forma se encuentra directamente relacionado con la competitividad del destino.

Por su parte Crouch y Ritchie (2000), afirman que la "competitividad de un destino turístico", está vinculada a la capacidad con la que un destino puede satisfacer las necesidades de los visitantes, en diversos aspectos de la experiencia turística. De ambas definiciones se puede concluir que la competitividad de un destino turístico debe satisfacer la calidad de vida de la población, de los actores que intervienen en la actividad turística y del turista, así como la rentabilidad económica de la actividad.

Otro axioma fundamental para el éxito en el mercado, es la valoración de la atractividad, para identificar las oportunidades y amenazas competitivas. Para medir el atractivo del mercado se necesita conocer su tamaño, su tendencia de crecimiento y otras variables que otorgan, no solo un amplio conocimiento del negocio, sino que brindan un enorme bagaje de información estratégica que redundará en oportunidades para incrementar la rentabilidad. Es por ello que se hace conveniente partir de un análisis de la competitividad ya que es medular tener la capacidad para configurar a través de una gestión eficiente de los recursos disponibles y del desarrollo de ciertas estrategias, una oferta de productos turísticos que sea atractiva para una cuota significativa de mercado, en rivalidad con otros competidores en un mercado abierto. (Dwyer y Kim, 2003)

El turismo para Cuba es un sector priorizado y el destino Cuba es uno de los destinos en desarrollo que va ganando significancia en el mercado mundial como los demuestran las cifras ascendentes de visitantes que recibe el país cada año, sin embargo, la necesaria dinamización de la economía exige el perfeccionamiento de su gestión sobre el proceso de implementación de los Lineamientos de la Política Económica y Social del PCC y la Revolución para el período 2017 - 2021; los cuales orientan que la actividad turística deberá tener un crecimiento acelerado que garantice la sostenibilidad y dinamice la economía, incrementando la competitividad de Cuba en los mercados turísticos, y diversificando las ofertas (L-209, L-210, PCC, 2017). Esto exige una acertada toma de decisiones, con la valoración de las potencialidades actuales y futuras del destino en un entorno marcado por una intensa competencia. 
Es por ello que se hace necesario el uso de herramientas que soporten una correcta planificación estratégica y brinden a los analistas información útil para la toma de decisiones. A esta situación se le añade la necesidad de desarrollar nuevas modalidades en el país para diversificar la oferta turística existente.

En la bibliografía consultada no se encontró una herramienta que vincule la competitividad del destino, y la atractividad del mismo evaluada en un mercado específico, con modalidad turística, facilitando de esta forma la toma de decisiones sobre una base científica sobre la introducción de una modalidad en el destino, es por ello que surge el siguiente, Problema de investigación: ¿Cómo pudiese relacionarse mediante una herramienta la atractividad del mercado, la competitividad del destino y la introducción de una modalidad turística?, planteándose como Hipótesis: La contextualización de la matriz de atractividad-competitividad en función de las modalidades turísticas facilitará el estudio de la relación de estas variables. Con el objetivo de contextualizar la matriz de atractividad-competitividad en función de las modalidades turísticas para su utilización como herramienta de planificación estratégica, se analizó la metodología para la conformación de la matriz de atractividadcompetitividad y las decisiones estratégicas a tener en cuenta en cada uno de los cuadrantes que la conforman. Se estudiaron las variables principales de la matriz original: la competitividad del destino y la atractividad del mercado, para establecer una siguiente etapa de análisis de la variable a incorporar, modalidad turística. Para ello se consultó a expertos en el tema, obteniendo los principales atributos que inciden en el potencial de desarrollo de las modalidades turísticas. Se aplicaron métodos estadísticos y matemáticos para la elaboración y conformación de la matriz resultante, la cual servirá de apoyo a analistas e investigadores en la planeación estratégica de destinos turísticos para la introducción de nuevas modalidades.

\section{Métodos.}

La metodología seleccionada para la presente investigación se sustentó en el diseño de una trayectoria compuesta por tres (III) fases:

Fase I. Establecimiento de los fundamentos teóricos y metodológicos de la investigación: El objetivo de esta fase fue en un primer momento establecer criterios, los enfoques fundamentales y las bases conceptuales que sustenten la base teórica de la investigación. Para dar cumplimiento al mismo se utilizaron diferentes métodos científicos de carácter teórico destacándose el método Histórico - Lógico, el cual permitió realizar el estudio de la información relacionada con los planteamientos de diferentes autores en el tiempo, acerca del tema de investigación y el análisis de la evolución histórica de los fenómenos que sustentan la temática tratada. Se emplearon, además, los métodos de Análisis y Síntesis e Inductivo Deductivo, los que permitieron arribar a conclusiones mediante generalizaciones derivadas del análisis y la deducción de los resultados particulares obtenidos y de la interpretación integral de la información revisada. Los resultados de esta fase se muestran en el informe general de la investigación.

Fase II. Estudio de la matriz de atractividad-competitividad: Esta fase tuvo como objetivo identificar las principales características de la matriz de atractividadcompetitividad, sus antecedentes, usos, limitaciones y la metodología para su elaboración. Empleándose como en la fase anterior los métodos de Análisis y Síntesis e Inductivo- 
Deductivo, los que permitieron arribar a conclusiones mediante generalizaciones derivadas del análisis y la deducción de los resultados particulares obtenidos y de la interpretación integral de la información revisada. Auxiliándose además de gráficos y tablas para la presentación más clara de la información obtenida.

Fase III. Contextualización de la matriz en función de la variable modalidad turística: El objetivo de esta fase es contextualizar la matriz de atractividadcompetitividad-modalidad, para ello se realizó una investigación de campo, con el objetivo de diseñar instrumentos y herramientas investigativas para conocer la percepción de los expertos con relación al tema.

a) Elaboración del instrumento cuestionario. El cuestionario se empleó en el método de consulta a expertos. Un primer cuestionario depuró, bajo criterio de expertos, los principales atributos para medir el potencial de desarrollo de una modalidad en un destino. Para la selección de los principales atributos se determinó un nivel de importancia del $60 \%$ y mayor que éste.

b) Test de cuestionario. Para este paso se utilizó un conjunto de preguntas que conformaban el futuro cuestionario a aplicar, y que responden a los objetivos específicos de la investigación. Las mismas fueron sometidas a análisis riguroso de un grupo de profesores y especialistas, que además de opinar sobre cada una de las preguntas y su contenido, colaboraron en la forma de enfoque y conclusión de éstas. Todos ellos vinculados a la temática abordada, procedentes de la Escuela de Altos Estudios de Hotelería y Turismo (EAEHT), y de la Facultad de Turismo de la Universidad de La Habana. Finalmente quedó elaborado y testeado el cuestionario. Este paso se efectuó antes de realizar las entrevistas a expertos.

c) Diseño del método de expertos. Las entrevistas fueron diseñadas con apoyo en la versión final del cuestionario, con vistas a identificar el conocimiento y percepción de los distintos atributos que influyen en el potencial de desarrollo de una modalidad turística en un destino determinado. Para ello se contó con el apoyo de un total de 7 expertos.

d) Captura, procesamiento y análisis. Una vez aplicadas las encuestas, se realizaron las funciones de captura, edición y tabulación de los resultados en bases de datos (Excel). La representación de la matriz se determinó atendiendo a criterios matemáticos para la ubicación de coordenadas en el espacio y del principio de multiplicidad para predecir las opciones de comportamiento de las variables, este proceso contó con la supervisión de la Licenciada en Ciencias Matemáticas Lucía Rosales Pitaluga.

\section{Resultados}

En el momento de análisis con los expertos se les solicitó que a su juicio determinaran los principales atributos para evaluar el potencial de desarrollo de una modalidad turística en un destino. La identificación de los mismos sirvió de guía para la inclusión de la variable modalidad turística en la matriz de atractividad- competitividad.

Tabla 1. Atributos resultantes para medir el potencial de desarrollo de las modalidades turísticas en un destino determinado. 


\begin{tabular}{|c|l|}
\hline $\begin{array}{c}\text { Grado de } \\
\text { importancia }\end{array}$ & \multicolumn{1}{c|}{ Atributos } \\
\hline 1 & Recursos y atractivos turísticos exclusivos para la modalidad \\
\hline 2 & Requerimientos de inversión \\
\hline 3 & $\begin{array}{l}\text { Existencia de sistemas de capacitación y formación del personal en } \\
\text { su totalidad para la modalidad }\end{array}$ \\
\hline 4 & Homogeneidad intracluster \\
\hline 5 & Heterogeneidad intercluster \\
\hline 6 & Marco regulatorio y presiones externas \\
\hline
\end{tabular}

Fuente: Elaboración propia.

La tabla anterior muestra los 6 atributos resultantes del método de expertos que influyen a su juicio en el potencial desarrollo de una modalidad turística en un destino determinado, los cuales son los recursos y atractivos turísticos de la modalidad, los requerimientos de inversión, la existencia de sistemas de capacitación y formación del personal en su totalidad para la modalidad, la homogeneidad intracluster, la heterogeneidad intercluster y el marco regulatorio y las presiones externas. Cada uno de ellos será analizado en el acápite discusión.

Tabla 2. Valores de la variable modalidades turísticas

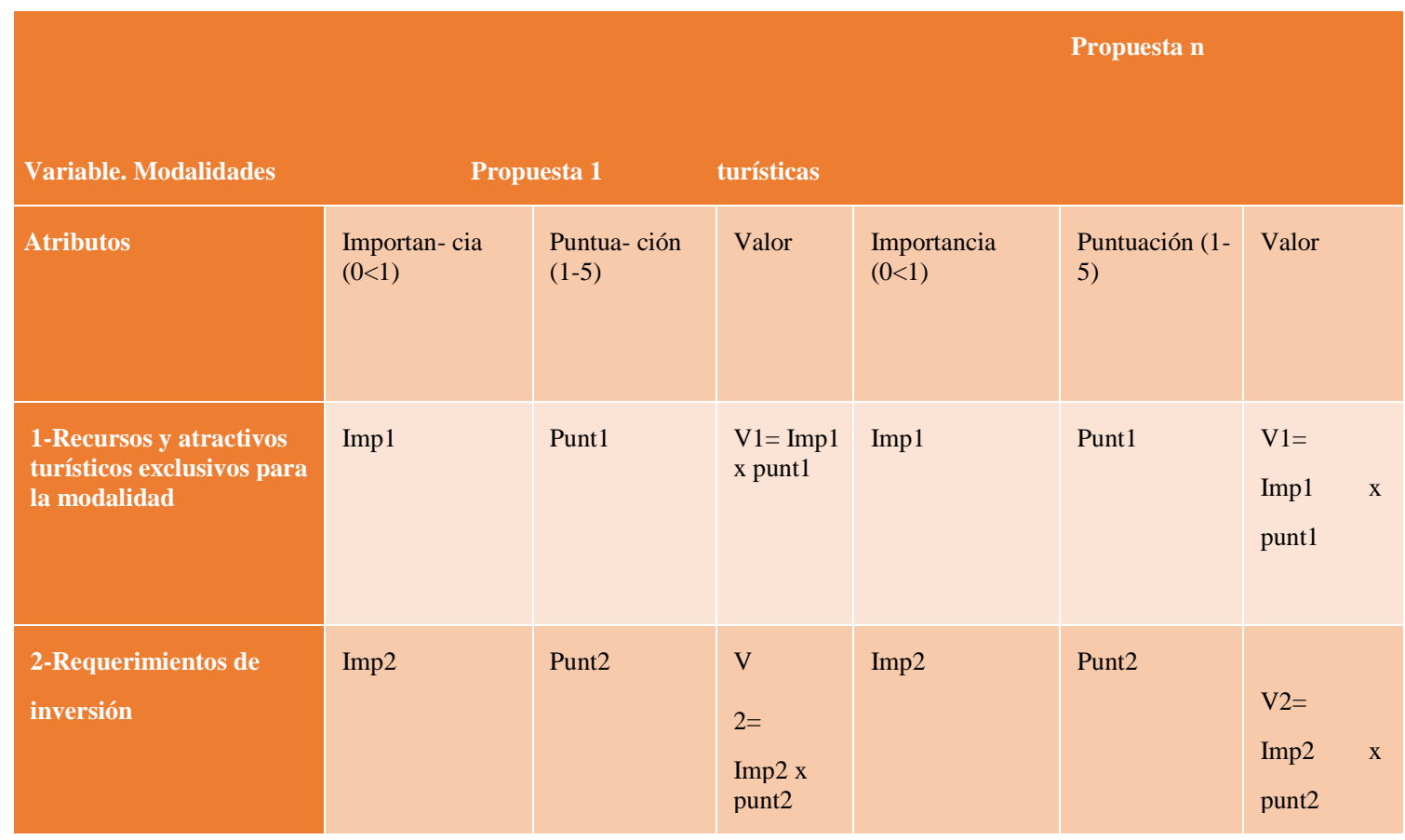




\begin{tabular}{|c|c|c|c|c|c|c|}
\hline $\begin{array}{l}\text { 3-Existencia de sistemas } \\
\text { de capacitación y } \\
\text { formación del personal en } \\
\text { su totalidad para la } \\
\text { modalidad }\end{array}$ & Imp3 & Punt3 & $\begin{array}{l}\mathrm{V} \\
3= \\
\text { Imp3 } \mathrm{x} \\
\text { punt3 }\end{array}$ & Imp3 & Punt3 & $\begin{array}{l}\text { V3= } \\
\text { Imp3 } \quad x \\
\text { punt3 }\end{array}$ \\
\hline $\begin{array}{l}\text { 4-Homogeneidad } \\
\text { intracluster }\end{array}$ & Imp4 & Punt4 & $\begin{array}{l}\mathrm{V} \\
4= \\
\text { Imp4 } \mathrm{x} \\
\text { punt } 4\end{array}$ & Imp4 & Punt4 & $\begin{array}{l}\text { V4= } \\
\text { Imp4 } \quad \mathrm{x} \\
\text { punt4 }\end{array}$ \\
\hline $\begin{array}{l}\text { 5-Heterogeneidad } \\
\text { intercluster }\end{array}$ & Imp5 & Punt5 & $\begin{array}{l}\mathrm{V} \\
5= \\
\text { Imp5 } \mathrm{x} \\
\text { punt5 }\end{array}$ & Imp5 & Punt5 & $\begin{array}{l}\text { V5= Imp5 } \\
\text { punt5 }\end{array}$ \\
\hline $\begin{array}{l}\text { 6-Marco regulatorio y } \\
\text { presiones externas }\end{array}$ & Imp6 & Punt6 & $\begin{array}{l}\mathrm{V} \\
6= \\
\text { Imp6 } \mathrm{x} \\
\text { punt6 }\end{array}$ & Imp6 & Punt6 & $\begin{array}{l}\text { V6= Imp6 } \\
\text { punt6 }\end{array}$ \\
\hline total & & & $\begin{array}{l}\Sigma(\mathrm{V} 1- \\
\mathrm{V} 6)\end{array}$ & & & $\begin{array}{l}\Sigma(\mathrm{V} 1- \\
\mathrm{V} 6)\end{array}$ \\
\hline Valor de Z & & & $\mathrm{Z} 1$ & & & $\mathrm{Zn}$ \\
\hline
\end{tabular}

Fuente. Elaboración propia

Una vez representadas las tablas que modelan el comportamiento de las variables, competitividad del destino, atractividad del mercado y modalidad turística, se procede a la representación gráfica de los valores obtenidos para facilitar el análisis de los resultados.

En la Figura 1 se muestra una posible representación del trabajo descrito, téngase en cuenta que las variables en cuestión presentan valores positivos de ahí que la representación de los datos tendrá lugar siempre en el octante limitado por los semiejes positivos.

Figura 1. Cuadrantes de posición de las variables.

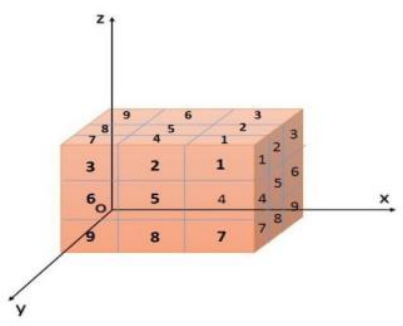

Fuente. Elaboración propia 


\section{Discusión.}

-Los recursos y atractivos turísticos exclusivos para la modalidad: Este atributo hace referencia a los recursos y atractivos existentes en el destino que tributan directamente a la modalidad que se esté evaluando. El analista debe por tanto realizar un estudio de los recursos y atractivos del destino y valorar cuál o cuáles de ellos son exclusivos de la modalidad turística que se propone. El valor a representar en la tabla dependerá de la razón entre el total de recursos y atractivos estudiados y los que son exclusivos de la modalidad entre los mismos. Para minimizar la subjetividad de la matriz en este atributo, se propone que si se cuenta con más del $50 \%$ de los recursos y atractivos, la propuesta es fuerte o alta, de $40-20 \%$ medio, y menor de un $20 \%$ se considera débil o baja.

-Los requerimientos de inversión: Con relación a este atributo el analista debe estimar los posibles requerimientos de inversión para el desarrollo de la modalidad en el destino, el valor a colocar en la tabla será inversamente proporcional a este resultado, o sea a menor requerimiento de inversión en la modalidad más fuerte o alta será considerada la propuesta.

\section{- La existencia de sistemas de capacitación y formación del personal en su totalidad para la modalidad:}

Satisfacer las expectativas de los clientes es esencial para cualquier organización. Además de centrarse en aspectos de administración interna, los directivos también deben satisfacer los requerimientos del cliente en cuanto a calidad, innovación, variedad y sensibilidad. Estos estándares requieren que las organizaciones ajusten sus procesos con las necesidades de los clientes en forma constante. Para integrar capital humano en las organizaciones, los directivos deben comenzar por desarrollar estrategias a fin de asegurar conocimiento, habilidades y experiencia superiores en su fuerza de trabajo. Los programas para definir puestos se centran en identificar, reclutar y contratar el mejor y más brillante talento disponible. Los programas de capacitación complementan estas prácticas de definición de puestos para mejorar las competencias laborales. Por tanto, este atributo, mide precisamente la existencia o no de estos sistemas de capacitación en concordancia con la modalidad y el segmento de la demanda que se esté evaluando.

-La homogeneidad intracluster y la heterogeneidad intercluster: El análisis de estos dos atributos responde directamente a la aplicación de técnicas de análisis clúster de ahí que su estudio y evaluación sea más conveniente realizarlo de manera simultánea. Comenzar aclarando que las aplicaciones del análisis clúster al marketing son múltiples, aunque, lógicamente, el campo donde mayores niveles de aplicación alcanza y donde más beneficios ofrece es el de la segmentación de mercados. También se ha utilizado en la identificación de hábitos de compra, de grupos de productos competitivos, valoración de productos de competidores o identificación de localidades que serán usadas posteriormente como mercados de prueba. En los últimos años, se ha revelado como una de las técnicas más utilizadas en el análisis de grupos estratégicos y en el desarrollo de tipologías estratégicas, casi siempre en combinación con otras técnicas multivariantes, como el análisis factorial y el análisis de la varianza. (Gómez, 2002)

Según la autora Gómez, (2002) el análisis clúster, usualmente consiste en dos pasos: El primero es calcular la proximidad, este refleja la similitud entre los elementos que se están 
investigando, el segundo consiste en la elaboración de los conglomerados formado por estos grupos homogéneos identificados anteriormente, de acuerdo a algoritmos matemáticos específicos. El objetivo es encontrar elementos en los clúster que sean lo más homogéneos posible, mientras que los elementos situados en clústeres diferentes sean lo más heterogéneo posible. La solución deseada se compone entre una alta heterogeneidad entre los distintos conglomerados y una alta homogeneidad dentro de los mismos.

Como primera medida, se han determinado una serie de puntos esenciales por los que cualquier investigador debe optar al aplicar esta técnica. Al no existir un consenso sobre qué elección es la mejor en algunas ocasiones, el investigador debe analizar las fuerzas o debilidades de un determinado enfoque y elegir aquel que resulte más conveniente a la hora de aportar objetividad a la investigación que desarrolla. Estos puntos esenciales al aplicar el análisis clúster son: la selección de variables, los algoritmos o métodos de agrupación, la determinación del número de grupos y la validación de los resultados.

-El marco regulatorio y las presiones externas: Cuando se analice este atributo se debe delimitar el entorno institucional que influye sobre la modalidad y el destino para luego analizar la influencia de las presiones institucionales sobre ellos. En dependencia del grado de impacto o del nivel de influencia que estas tengan sobre la propuesta evaluada, serán colocados los datos en la tabla que mide la variable modalidad turística. Solo resta entonces proseguir a la contextualización de la matriz en cuestión, para ello se comenzará mostrando los modelos de las tablas con las que se describen las variables atractividad del mercado, competitividad del destino y modalidad turística.

Después de representados los datos en la matriz, y teniendo en cuenta el principio de multiplicidad se pueden predecir 27 posibles comportamientos para las tres variables, en función de las escalas de valores que se emplearan en esta metodología, tal que de 02 el comportamiento de la variable es considerado bajo o débil, de 2-4 medio, y mayor a 4 alto o fuerte. Luego se deben establecer las acciones en dependencia de estos comportamientos.

\section{Estrategias}

-Requiere esfuerzo en inversión y crecimiento. Estas decisiones se corresponden a las propuestas ubicadas en el primer cuadrante. Las propuestas situadas en esta zona ostentan la posición de liderazgo. Deben tener por tanto prioridad en cuanto a la asignación de recursos con el fin de maximizar las inmejorables perspectivas que poseen y sostener sus puntos fuertes.

-Inversión y crecimiento selectivo. Estas decisiones se corresponden a las propuestas ubicadas en el segundo y cuarto cuadrante. Dado el carácter intermedio de estas propuestas, se debe trabajar en función de identificar y solventar los puntos débiles.

-Selección oportunista. Estas decisiones se corresponden a las propuestas ubicadas en el tercer cuadrante. Las propuestas analizadas aquí podrían llegar a ser las más prometedoras. La empresa debe seleccionar cual variable presenta un potencial de crecimiento mayor para enfocarse en ella y mejorar esta posición. 
-Selectividad. Estas decisiones se corresponden a las propuestas ubicadas en el quinto cuadrante. Es necesario ser muy prudentes con ellas pues todas las variables en este cuadrante presentan valores medios, se aconseja invertir selectivamente y especializarse en el mercado que esté al menos, estabilizado en el destino con la modalidad analizada.

-Selección protectora. Estas decisiones se corresponden a las propuestas ubicadas en el séptimo cuadrante. Las propuestas aquí analizadas o bien presentan un mercado inestable o en presencia de una modalidad poco desarrollada, de ahí que el enfoque debe hacerse en función de mejor estas dos variables.

- Cosechar a través de tácticas invisibles. Estas decisiones se corresponden a las propuestas ubicadas en el sexto cuadrante. Para las cuales se mantiene la propuesta en los niveles mínimos de coste con la esperanza de obtener la mayor rentabilidad a corto plazo.

- Cosechar a través de tácticas visibles. Estas decisiones se corresponden a las propuestas ubicadas en el octavo cuadrante. Se poseen ciertas ventajas. Hay que proteger la posición de aquellas propuestas ubicadas en segmentos rentables.

-Desinversión rápida. Estas decisiones se corresponden a las propuestas ubicadas en el noveno cuadrante. En esta situación la propuesta no se puede desarrollar pues el comportamiento de las tres variables es bajo lo que implica un replanteamiento de la misma.

\section{Conclusiones}

El potencial de desarrollo de una modalidad turística, puede evaluarse a través de: los recursos y atractivos turísticos exclusivos de la modalidad, los requerimientos de inversión, la existencia de sistemas de capacitación y formación del personal en su totalidad para la modalidad, la homogeneidad intracluster, la heterogeneidad intercluster y el marco regulatorio y las presiones externas.

$>$ La propuesta de matriz de atractividad-competitividad-modalidad, aunque puede considerase subjetiva pues depende en gran medida de la opinión y juicio de los analistas, acompañada de otras herramientas podrá contribuir a la planeación estratégica de destinos turísticos para la introducción de nuevas modalidades. 


\section{Referencias Bibliográficas}

Barroso, María y Flores, David. (2006). La competitividad internacional de los destinos turísticos: del enfoque macroeconómico al enfoque estratégico. Cuadernos De Turismo, (17), 7-24.

Dwyer L. and Kim, C. (2003). "Destination Competitiveness: Determinants and Indicators. Current Issues in Tourism, Volume 6, Issue 5 October 2003, pages 369 $-414$

Crouch, G. I., \& Ritchie, B. J. R. (2000). Tourism, competitiveness, and societal prosperity. Journal of Business Research, 44(3), 137-152.

Lineamientos del séptimo Congreso de la Política Económica y Social del PCC y la Revolución para el período 2017 - 2021

Agudo, A. y Fernández, J.M. (1994), “Métodos de segmentación basados en la aplicación combinada de técnicas estadísticas", VI Encuentro de Profesores Universitarios de Marketing, San Sebastián, pp.99-107.

Bosch Campubrí, R.,Marco, L. P.,Cabado, J. S., \& Riera, F. V. (1998).Turismo e meior ambiente. Madrid:Centro de Estudios

Ramon Areces. Boston, Massachusetts: Harvard Business School Press. Modelos de Competitividad para Destinos Turísticos en el Marco de la Sostenibilidad RAC, Curitiba, v. 12, n. 3, p. 789-809, Jul./Set. 2008809

Cooper, C. (2002): "Sustainability and tourism visions", VII Congreso Internacional del CLAD sobre la Reforma del Estado y de la Administración Pública, Lisboa, 8-11 octubre, 2002.

Crouch, G. I., \& Ritchie, J. R. B. (1999): "Tourism competitiveness and societal prosperity".Journal of Business Research, 44(3), 137-152.

Dwyer L. y Kim C.W. (2001): Destination Competitiveness: Development of a Model with Application to Australia and the Republic of Korea. Report prepared for Department of Industry Science and Resources, Australia and Korea Tourism research Institute, Ministry of Tourism, October.

\section{\Ciencia}




\section{Para citar el artículo indexado.}

Llanes D. \& Bulnes D. (2017). Propuesta de la matriz de atractividad - competitividad en función de las modalidades turísticas. Revista electrónica Explorador Digital 1(2), 3242. Recuperado desde:

http://cienciadigital.org/revistacienciadigital2/index.php/exploradordigital/article/view/320/7 $\underline{32}$

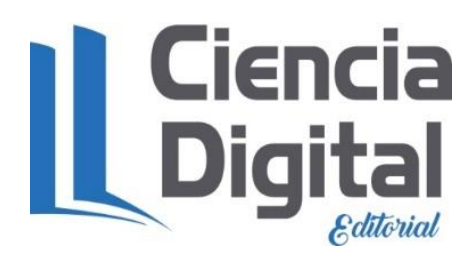

El artículo que se publica es de exclusiva responsabilidad de los autores y no necesariamente reflejan el pensamiento de la Revista Explorador Digital.

El articulo queda en propiedad de la revista y, por tanto, su publicación parcial y/o total en otro medio tiene que ser autorizado por el director o editor de la Revista Explorador Digital.
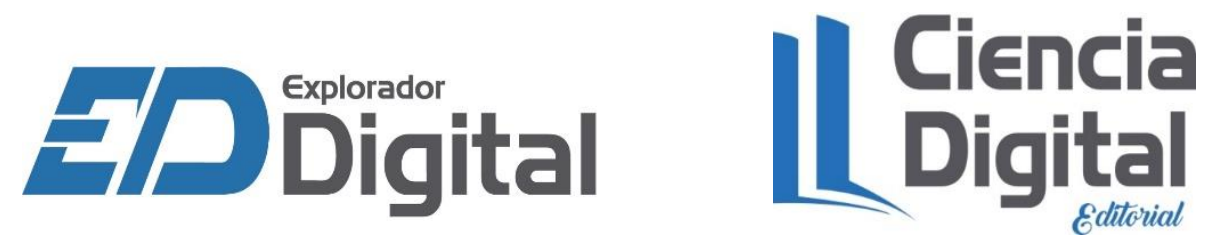